
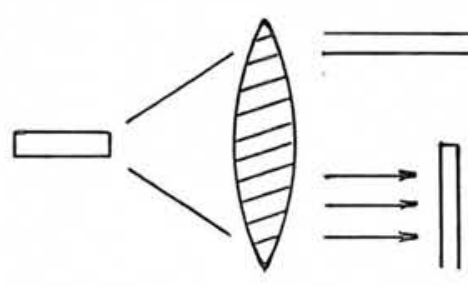

laser $s\left(x_{1}, y_{1}\right)$

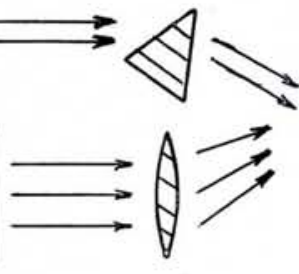

$\left.y_{1}\right)$
Fig. 4 Technique for preparing a Vanderl

ing is differentiation. To differentiate a function $f(x)$ one has to determine the filter function $F$, whose convolution with the function gives the derivative $d f / d x$.

Such a mask, called a differentiator, has an amplitude transmittance which varies linearly with distance. Higher order derivatives are obtained with the aid of transmittances varying as the power of the desired derivative. Also mixed higher order derivatives such as $\delta^{2} f / \delta x \delta y$ can be obtained, and even an integrator where the transmittance varies as the reciprocal of the distance in the transform plane. From this it follows that all the classical operators, for example the Laplacian operator or transforms, e.g. the Hilbert transform can be effected. It is important to note that the operation can be carried out in one step. As a result there is not the degradation of accuracy that occurs in electronic computing with successive processes. In the case of higher order differentiation this is particularly marked as also is the insensitivity to high slopes approaching singularities.

Finally it should be noted that besides these computing capabilities, optical devices have tremendous storage capacities, and these can be digitized. From this aspect alone, although the study of optical data processing is relatively in its infancy, one can be sure that in the computers of tomorrow, optical components will have an important place.

\section{Basic References}

1. ABBE, E., Archiv. Mikroskopische Anat. 9. (1873) 413

2. DE VELIS, J. and REYNOLDS, G., Theory and Applications of Holography (Addison-Wesley) 1967

3. PRESTON, K., Coherent Optical Computers (McGraw-Hill, New York) 1972

4. LIPSON, H.S., Optical Transforms (Academic Press) 1972

5. KOCK, W., Engineering Applications of Lasers and Holography (Plenum Press) 1975

6. GOODMAN, J., Introduction to Fourier Optics (McGraw-Hill, New York) 1968

7. GABOR, D., "Les Transformations de I'Information en Optique" Optica Acta 13 (1966) 4

8. PERNICK, B. and DE CARLO, J., "Optimum Imaging Method for Optical Data Processors" Applied Optics 16 (1977) 1

\title{
Tokamak Reactors for
}

\section{Break-even H. Knœpfel, Frascati}

\section{A critical study of the near-term fusion reactor programme}

Fig. 1 Schematic view of a Tokamak. The air-core transformer induces the current $I_{p}$ that heats the plasma and produces the magnetic field $B_{P}$ necessary for equilibrium and stability. The compact toroidal magnet is typical for high field $\left(B_{T} \approx 10 \mathrm{~T}\right.$ ) devices. Not shown are coils producing a vertical field $B_{V}$ for centring the plasma colum.

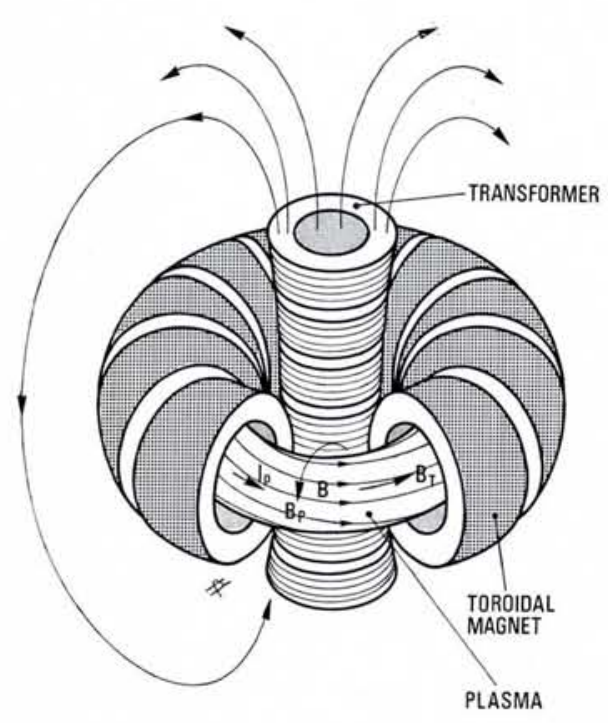

At a time when discussions on the definition of the Joint European Torus programme (JET) are still going on, it may be useful and interesting to look back for a moment to the Course held in Erice in September 1976 on Tokamak Reactors for Break even - A Critical Study of the Near Term Fusion Reactor Program. The title refers to Tokamak devices (Fig. 1) in which the thermonuclear energy produced from a deuterium-tritium plasma, represents a substantial fraction of the energy required to sustain the machine cycle and heat the plasma. The discussion at Erice was thus centred on the steps that should follow JET; implicitly, it also provided a valuable contribution to the definition of the aims and prospects of a much needed near-to-medium term European fusion programme. Such a definition could be of much help to Euratom, which is in charge of coordinating the West European fusion effort.

The present effort in the field of controlled thermonuclear fusion (Table 1) represents a substantial new experience for all those concerned with research and development pro-
(CNEN lonized Gas Laboratory)

grammes. In fusion, we find an intriguing mixture of fundamental and applied research, and of technological developments as well; moreover, the further the work proceeds, the more these $R$ \& D programmes (all equally important) will have to match the ultimate promise of fusion as a new energy source. The unusual but necessary variety of interweaved approaches and motivations is what makes fusion research to many so confusing - and, at the same time exciting; it also makes it so essentially different from other major technological and scientific adventures, such as the exploration of space, or the development of nuclear breeders,

\section{Table I: Controlled Fusion 1977}

1. Effort required to reach reactor stage may be larger than the NASA Apollo Programme.

2 Fusion has now entered into competition for funds and support with other important R \& D programmes.

3. World effort in magnetic fusion is supported by more than 3000 professionals and an estimated $700 \mathrm{M} \mathrm{\$ ;} \mathrm{more} \mathrm{than}$ $2 / 3$ are directly or indirectly for the Tokamak line.

4. There is as yet no clear indication on the scientific feasibility. 
or research in high energy physics, where the technical component is much better separated from - or even independent of - the physics programme.

Among the many requirements imposed on a thermonuclear magnetoplasma, are two that are fundamental to the practical application of fusion: a) A positive power balance is required; in a plasma with the energy confinement time $\tau_{E}[=$ total plasma energy)/(ohmic heating power)] this condition can be expressed approximately in the form:

$$
\begin{aligned}
Q= & \frac{\text { power out }}{\text { power in }} \\
& \approx \frac{n^{2}(\sigma v) W_{\mathrm{f}}}{n k T / \tau_{\mathrm{E}}+P_{\mathrm{rad}}}>1
\end{aligned}
$$

where $n$ is density, $n^{2}(\sigma v)$ is the mean fusion reaction rate, $W_{f}$ the nuclear fusion energy, $n k T$ the thermal plasma energy and $P_{\text {rad }}$ the power loss through radiative phenomena. When the latter term is negligible, then $Q \approx n \tau_{E}(\sigma V / T) W_{f}$, where for a deuterium-tritium plasma $(\sigma \mathrm{V} / T)$ has a maximum around $T \sim 25 \mathrm{keV}$.

b) A high power density is required for economic energy production:

$$
p=n^{2}(\sigma v) W_{f} \propto \beta^{2} B^{4}\left(\sigma v / T^{2}\right)
$$

Notice that the (toroidal) field $B$ is limited at different levels by technical restraints (see Sect. 3) and the parameter $\beta \equiv$ (plasma energy)/(magnetic energy) by plasma physical effects (see next Section). Nevertheless, typical power densities of $p \simeq 10 \mathrm{MW} / \mathrm{m}^{3}$ would be conceivable (compared with $300 \mathrm{MW} / \mathrm{m}^{3}$ in a fast breeder reactor). There is, however, a fundamental upper limit of $p$ defined by the fusion reactor surface constraint. In practice, the (neutron) power flux through the first plasma container wall is limited by material properties (structural damage) to less than e.g. $5 \mathrm{MW} / \mathrm{m}^{2}$ for stainless steel liners. For a reactor with plasma and liner radii of 3 and $3.5 \mathrm{~m}$ respectively, the mean power density would thus be limited to $4 \mathrm{MW} / \mathrm{m}^{3}$.

Table II: Current Experimental Status of Magnetic Fusion Systems

\begin{tabular}{|l|c|c|c|c|}
\hline & $\begin{array}{c}n \tau_{\mathrm{E}} \\
\left(\mathrm{cm}^{-3} \mathrm{~s}\right)\end{array}$ & $\begin{array}{c}\beta \\
(\%)\end{array}$ & $\begin{array}{c}T_{\mathrm{i}} \\
(\mathrm{keV})\end{array}$ & $Z_{\text {eff }}$ \\
\hline $\begin{array}{l}\text { Fusion } \\
\text { Feasibi- } \\
\text { lity }\end{array}$ & $10^{14}$ & 10 & 10 & $1-2$ \\
\hline $\begin{array}{l}\text { Tokamaks } \\
\text { Mirrors }\end{array}$ & $10^{13}$ & 2 & 2 & $1-10$ \\
Pinches & $10^{11}$ & $50-100$ & 10 & 1 \\
\hline 11 & $50-100$ & 4 & $1 ?$ \\
\hline
\end{tabular}

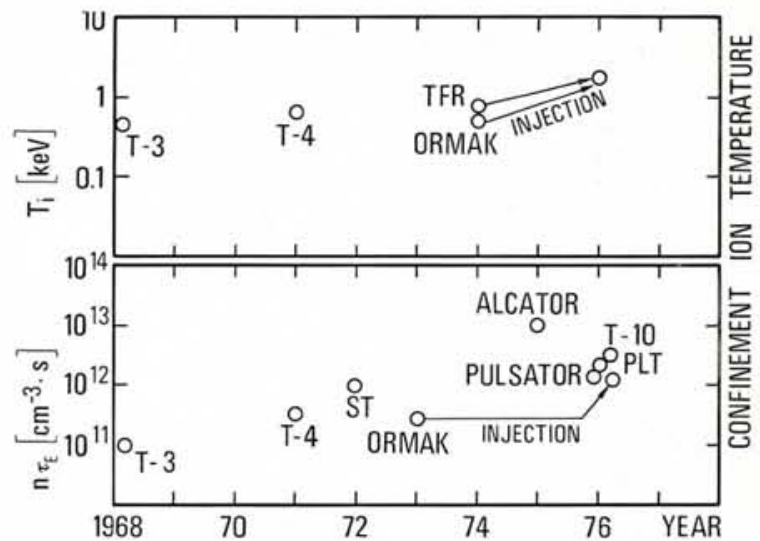

Fig. 2 Larger dimensions, higher magnetic fields and neutral injection heating have improved Tokamak plasmas over the past 10 years. The locations of this selection of Tokamaks, major $\left(R_{0}\right)$ and minor (a) radii, and experimental toroidal fields $\left(B_{T}\right)$ on the axis are as follows:

T-3, Moscow (100/15 cm, $2.5 \mathrm{~T}) ; \mathrm{T}-4$, Moscow $(100 / 17 \mathrm{~cm} ; 4 \mathrm{~T})$; ST, Princeton $(109 / 13 \mathrm{~cm} ; 4 \mathrm{~T})$; ORMAK, Oak Ridge $(80 / 23 \mathrm{~cm} ; 2.5 \mathrm{~T})$; ALCATOR, MIT (54/10 cm ; $7 \mathrm{~T})$; TFR, Fontenay-aux-Roses $(98 / 20 \mathrm{~cm} ; 5 \mathrm{~T})$; PULSATOR, Garching (70/11 cm; $2.7 \mathrm{~T}) ;$ PLT, Princeton (130/45 cm; $3.5 \mathrm{~T}) ; T-10$, Moscow $(150 / 40 \mathrm{~cm} ; 3.5 \mathrm{~T})$.

\section{Plasma physical aspects}

From the previous expression (1) the following typical set of minimal parameter conditions can be deduced to arrive at the necessary power gain in a deuterium-tritium plasma: $n \tau_{\mathrm{E}} \supseteq 10^{14} \mathrm{~cm}^{-3} \mathrm{~s}$ (Lawson criterion); $T_{\mathrm{i}}>6 \mathrm{keV} ; Z_{\text {eff }}<2$, where $Z_{\text {eff }}$ is the mean effective ion charge that reflects the impurity content. (For example: $Z_{\text {eff }}=1$ for a pure hydrogen plasma, but $Z_{\text {eff }}=2$ with $2 \%$ fully ionized oxygen impurity in it). In addition, if a realistic evaluation is made of the technical and economical constraints that limit the maximum magnetic field, expression (2) imposes, in practice, the condition $\beta \geqslant 10 \%$ for reactor applications.

These fundamental parameters have improved steadily in Tokamak plasmas over the past 10 years (Fig. 2), but probably not as much as many hoped (Table II). It is commonly accepted that further appreciable progress in understanding and in performance can be obtained only with larger and/or technologically more advanced devices, and with substantial new heating capabilities.

\section{a) High Power Densities $(\beta)$}

There are several methods by which, in principle, $\beta$ can be improved from the present $1-2 \%$ to above 5-10\%: elongating the cross section (as in JET); shaping the current profile (which also requires auxiliary heating); establishing a force-free current zone that surrounds the main plasma (as in toroidal pinches); applying the so-called flux conserving Tokamak scheme (as proposed by Oak Ridge). Numerical studies with two-dimensional magneto-hydrodynamic codes show, however, that it is difficult to reach values higher than about $5 \%$, because of MHD stability problems of ballooning modes. These instabilities are a consequence of the large density and magnetic field gradients in the outer equatorial regions of high $\beta$ discharges; they tend to make the pressure profiles across the whole discharge evolve into large bulges.

\section{b) Containment $\left(n \tau_{E}\right)$}

The increase of the $n \tau_{E}$ parameter over the past 20 years in various fusion experiments is very impressive: $10^{9} \mathrm{~cm}^{-3} \mathrm{~s}$ in $1955 ; 10^{11}$ in 1965; more than $10^{13}$ in 1976 ! It is thus tempting to speculate on future improvements in Tokamaks by matching experimental results with empirical scaling laws. For example, within the limited parameter range of (small) Tokamak experiments, the scaling $n \tau_{\mathrm{E}} \propto n^{2} \mathrm{a}^{2}$ holds fairly well, where $a$ is the minor toroidal plasma radius. By applying the conditions that express MHD equilibrium constraints, from this expression an upper limit can be obtained:

$$
\left(n \tau_{\mathrm{E}}\right)_{\max } \propto q^{-3.5} R_{\mathrm{o}}^{2} B^{4}\left(a / R_{\mathrm{o}}\right)^{4}
$$

$q$ is the so called safety factor: $q=4 \ldots 6$ in actual Tokamaks, and hopefully may approach 2 in future experiments. If the recent results of the two largest Tokamaks are taken into account, the scaling is somewhat less favourable, $n \tau_{E} \propto(n a)^{3 / 2} B$. In any case, the important temperature scaling of confinement is missing as it is difficult to determine from present experiments, because of the inherent coupling between confinement and ohmic heating in the Tokamak confi- 
guration (this restraint will be relaxed with the application of future large be emphasized, however, that scaling laws that are not based on a proven and comprehensive plasma model have little physical meaning and should not be used as crystal balls for reading the future of fusion.

\section{c) Heating $\left(T_{i}\right)$}

Up to now, neutral injection heating has been an outstanding success story (Fig. 3). In several machines, the auxiliary injected power is approaching the ohmic power (though at levels of only some hundreds of kilowatts), and the heating of ions has continued to behave neoclassically. The electron temperature, on the other hand, shows a puzzling reluctance to increase correspondingly; this behaviour is somewhat disturbing, but the experiments are still inconclusive and leave room for different explanations and for hopes of improvement. Electromagnetic wave heating, after about 20 years of benevolent attention, has now the opportunity to show its practical relevance in different experiments. For example, $5 \mathrm{MW}$ of ion cyclotron heating (typically in the 25 to $150 \mathrm{MHz}$ range) is foreseen for the PLT-Tokamak in Princeton.

\section{d) Impurities $\left(Z_{\text {eff }}\right)$}

In some experiments, impurities are apparently ejected by instabilities, or held outside the discharge by temperature gradients; high density discharges are surprisingly pure $\left(Z_{\text {eff }} \rightarrow 1\right)$. There is, as yet, no real understanding of the somewhat confusing dynamics of impurities but the situation is more promising than was the case even two years ago.

Main hopes rest on the use of divertors, on a careful choice of limiter auxiliary heating systems). It should

materials, on operation at higher densities and on the reduced surface to volume ratio of larger machines.

\section{Technologies and Systems}

The technological requirements of future large toroidal devices - at first, machines of the dimensions of JET and then especially the eventual Demonstration Reactor - are very substantial. On this general level there are at present two rather different attitudes to the development of systems and their required technologies.

Either they could evolve with experiments and new ones be added as the experiments slowly develop into the ultimate reactors, or systems specific to the future reactors could be studied now, in parallel with current experiments. The first method - which could be called the "evolutionary approach" - is probably the most economic in that no unnecessary system or technologies would be generated. However, current experiments not actually aimed at reactor conditions, could be a misleading guide for the development of systems and technologies.

The second method - the "planned approach" - has the advantage that the Reactor System Studies could help guide the next stage of the experiments and indicate future problems. Such studies have, in fact, highlighted certain technical problems, such as materials problems and plasma-wall interaction problems, which will take a long time to resolve and hence should be started in parallel with the present day confinement experiments in order to reduce the time to a fusion reactor. However, if there is no effective management, technological studies can easily grow out of control, thereby absorbing a

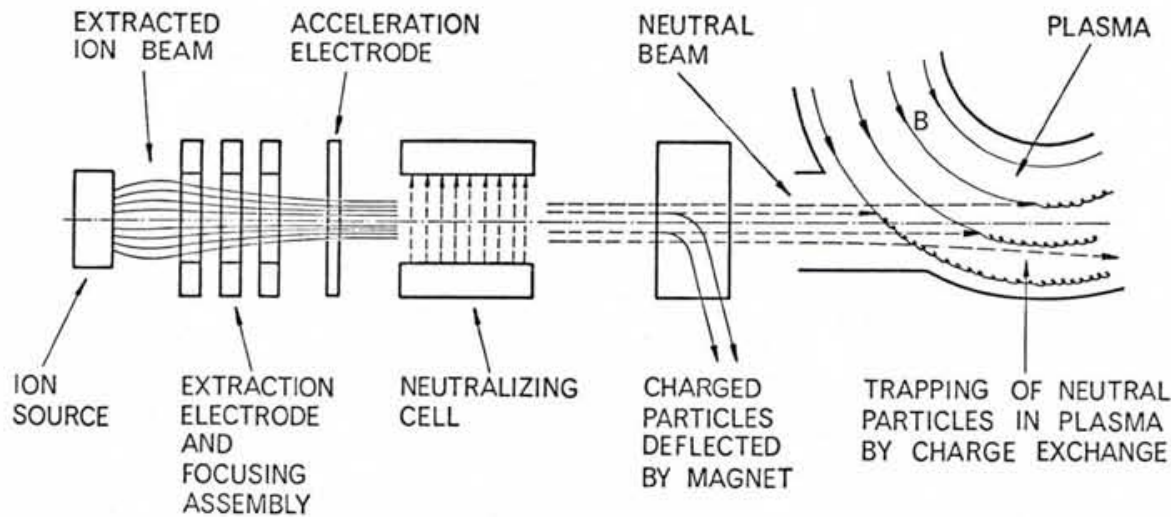

Fig. 3 Neutral beam injectors, as an auxiliary heating source, are contributing substantially to the progress of fusion. A single unit (built by Oak Ridge for PLT) will deliver to the plasma $1 \mathrm{MW}$ of neutrals at $40 \mathrm{keV}$. Similar units are being prepared by the European laboratories at Culham and Fontenay-aux-Roses. great deal of money at the expense of the experiments presently under way.

There are some specific technical systems that may be important for near term experiments, as well as the ultimate reactor. Typical examples are: neutral particle injectors (Fig. 3) and superconducting magnets. Some megawatts of neutral particles will be injected into the PLT Tokamak in Princeton towards the end of 1977.

A power of approximately $30 \mathrm{MW}$ of neutral injection heating is foreseen for the Tokamak Fusion Test Reactor $\left(R_{\mathrm{o}}=250 \mathrm{~cm}, a=85 \mathrm{~cm}, B_{\mathrm{T}}=5 \mathrm{~T}\right)$ which should become operational in 1982 in Princeton; for JET $\left(R_{\mathrm{o}}=300 \mathrm{~cm}, a=210 / 120 \mathrm{~cm}, B_{\mathrm{T}}=\right.$ $2.7 \mathrm{~T}$ ) one now requires, on the basis of scaling laws as given in Sect. $2 b$, an injected power of more than 25 MW. Such large heating systems represent a substantial investment, estimated to be $1.5-2 \mathrm{~S} / \mathrm{W}$ of neutral beam power.

The importance of magnet technology with respect to obtainable fusion plasmas is demonstrated directly by expression (2); on the other hand, there are the aspects and the limitations imposed by mechanical stresses and, particularly, by ohmic dissipation in the coils.

It is convenient to consider four different magnetic field ranges, each characterized by its particular problems and specific advantages. Notice that $B$ indicates here the magnetic field on the toroidal axis $\left(R=R_{\mathrm{o}}\right)$; due to the $1 / R$ dependence, the maximum toroidal field at the inner equatorial region of each coil is $R_{0} /\left(R_{0}-a\right)$ times larger (typically $70 \%$ larger).

a) $B<5 \mathrm{~T}$ : Conventional watercooled copper magnet, made typically of 16 to 32 single coils that give good access for auxiliary heating and diagnostics to the plasma container (e.g., DITE Tokamak at Culham or PLT at Princeton); possibility of using proven $\mathrm{NbTi}$ superconductors with partial or full cryostatic stabilization (e.g., T-7 and T-10M Tokamaks in Moscow, operation foreseen in 1978 and 1981).

b) $5 \mathrm{~T}<B<8 \mathrm{~T}$ : Mechanically advanced and reinforced copper magnets that still allow reasonably good access (e.g., TFR Tokamak at $6 \mathrm{~T}$ in Fontenay-aux-Roses); possibility of using high field A 15 superconductors such as $\mathrm{Nb}_{3} \mathrm{Sn}$.

c) $8 \mathrm{~T}<B<14 \mathrm{~T}$ : Compact magnet of the Bitter disk type at cryo- 
genic temperatures; limited access (e.g., Frascati Tokamak at $10 \mathrm{~T}$, or ALCATOR C designed for $14 \mathrm{~T}$ at MIT).

d) $14 \mathrm{~T}<\mathrm{B}$ : Compact one-turn toroidal solenoid; very poor access.

High magnetic fields allow (according to expression (2), Sect. 1) the operation of denser plasmas in more compact devices. In addition, so far the best results concerning $n \tau_{\mathrm{E}}$ and $Z_{\text {eff }}$ have been obtained in high field and high density operation. High field Tokamaks pose challenging technical problems, but represent today an interesting approach to fusion that is complementary to the low field and low density regimes contemplated up to now in larger devices (Table III). Since magnetic fusion reactors will, most probably, require superconducting magnets for economical reasons, the long range high field prospects concentrate on the magnetic field range $b$ ). On the other hand, devices operating in the range c) or d) may be very interesting in the intermediate phase, to demonstrate controlled ignition of a deuterium-tritium plasma, or as a material testing device.

\section{Conclusions}

Unless a substantial scientific breakthrough emerges in the next five years (which is not at all impossible) and unless the world-wide fusion technology effort is increased manyfold, it looks unlikely that a fusion demonstration reactor (at the level represented, for example, by the Phénix Reactor in the fast breeder programme) could be effectively working before the year 2000. In the next years it will be necessary to work out long- term programmes that are sufficiently flexible - and credible - that they can accommodate delays or fundamental reorientations in fusion research and development, even if that means shifting the target date of the demonstration reactor to the year 2010 or beyond. Medium-term programmes that give useful guidance on the choices open in fusion research, exist in the United States and are now particularly needed in Europe. In view of the future large prototypes and of the related costs and risks of the complex research and development programmes, medium-term planning on a broad international level is becoming ever more desirable, even necessary.

Discussions at the Erice meeting have shown that a majority of the scientific community is realistically aware of the many important implications of the future fusion research programmes. It was clear to all that magnetic fusion has now reached a level of political attention and funding (270 M S in 1978 in the U.S. alone) that a strong, competent and imaginative management has become necessary. Only gradually is the community becoming aware of the fact that fusion research and development, if it is finally to be carried through to success, will probably surpass in impact and in technological and financial dimensions, for example, the NASA Apollo Programme.

At the same time, there is a second aspect, namely the scientific and intellectual motivation for this activity that will make it possible to survive inevitable pauses or drawbacks during its progress. In fusion-oriented plasma research and development there is a challenge of new frontiers in physics, of new numerical and theoretical working methods, of sophisticated diagnostic systems and of advanced technologies that in its totality is hardly matched by any other scientific field of human endeavour.

Table III: Build Larger Tokamaks now? Yes, because :

1. Fusion reactors will necessarily be large (minor radius: $2-3 \mathrm{~m}$, major radius: $5-8 \mathrm{~m}$; the sooner one is confronted with the physics and technology of large systems, the better).

2. Alpha particles will be contained (the physics of thermonuclear heating can be studied)

3. General physical considerations and results from present Tokamak generation, suggest a favourable scaling with size.

4. Surface to volume ratio is reduced (the impurity influx, and thus heat losses, should be reduced).

5. Quality of magnetic topology may be improved.

6. Good access for injection heating and diagnostics is provided.

7. Solutions to important new technology problems (superconducting magnets?) will be sought.

No, because :

1. The outstanding physics questions (containment, heating, plasma beta, impurities) will barely be answered by 1985.

2. Within limited budgets, the priority must be given to basic physics problems.

3. The so-called scaling laws in support of larger devices are not based on sufficient scientific data (for example, temperature scalling in Tokamaks can be studied only with future injection heated devices).

4. No fusion concept can scientifically claim at present to be the most promising (a high field device, for example, may turn out to be more convenient).

\section{Waiting for JET}

Still no decision has been taken on the project to build the Joint European Torus, $J E T$, although it had been hoped that some solution might have been found at the meeting of the heads of State of western European countries that took place in London in June. Now it is hoped that something positive may emerge from the meeting of the Council of Foreign Ministers of the countries of the European Communities on September 20.

Wide publicity has been given to the problem of selecting a site with, at various times, the centres at Cadarache, Culham, Garching and Ispra being quoted. The site is not, however, the only decision that has to be taken. The Committee of Permanent Representatives of the Communities has to recommend a management structure for the Project and this also has been the subject of controversy. Everyone seems to be in agreement that JET is a project of great importance for the development of fusion and is vital for the promotion of the technology in western Europe but this has only served to accentuate the divergences and regretably the politics of collaboration have seemed to dominate the scientific imperatives.

A certain parallel exists between the present JET situation and that encountered with the CERN SPS in the latter part of the 1960's, but at CERN it proved possible to appoint without too much argument a project leader who was able then to catalyse the subsequent accord. There was also, of course, an existing single international site whereas, although Euratom has been successfully coordinating western European research on fusion devices over many years, there is as yet no single European centre devoted to fusion re search. JET has so far no project leader. but a design team leader in the person of Paul Redut. The remit of his team at Culham has been extended to cover the months of August and September but it seems unlikely that the members of this team will be prepared to continue on such a hand to mouth existence indefinitely.

In the meantime, the only large, new Tokamak presently being built in Europe is the ASDEX device in Garching near Munich shown in the photograph on the opposite page. 